\title{
Management of severe COPD exacerbations: focus on beclomethasone dipropionate/ formoterol/glycopyrronium bromide
}

This article was published in the following Dove Press journal: International Journal of COPD

\author{
Marco Mantero',2 \\ Dejan Radovanovic ${ }^{3}$ \\ Pierachille Santus ${ }^{3}$ \\ Francesco Blasi ${ }^{1,2}$ \\ 'Department of Pathophysiology \\ and Transplantation, University of \\ Milan, Milan, Italy; ${ }^{2}$ Internal Medicine \\ Department, Respiratory Unit \\ and Regional Adult Cystic Fibrosis \\ Center, IRCCS Fondazione Ca' \\ Granda Ospedale Policlinico, Milan, \\ Italy; ${ }^{3}$ Department of Biomedical and \\ Clinical Sciences (DIBIC), Pulmonary \\ Unit, University of Milan, Ospedale L. \\ Sacco, ASST Fatebenefratelli-Sacco, \\ Milan, Italy
}

\begin{abstract}
The major determinant of the decline in lung function, quality of life, and the increased mortality risk in patients with COPD is represented by severe acute exacerbations of the disease, that is, those requiring patients' hospitalization, constituting a substantial social and health care burden in terms of morbidity and medical resource utilization. Different long-term therapeutic strategies have been proposed so far in order to prevent and/or reduce the clinical and social impact of these events, the majority of which were extrapolated from trials initially focused on the effect of long-acting muscarinic antagonist and subsequently on the efficacy of long-acting $\beta 2$-agonists in combination or not with inhaled corticosteroids. The option to employ all three classes of molecules combined, despite the limited amount of evidence in our possession, represents a choice currently proposed by international guidelines; however, current recommendations are often based mainly on observational studies or on the results of secondary outcomes in randomized controlled trials. The present narrative review evaluates the available trials that investigated the efficacy of inhaled therapy to prevent COPD exacerbations and especially severe ones, with a particular focus on beclomethasone dipropionate/formoterol/glycopyrronium bromide fixed dose combination, which is the first treatment that comprises all the three drug classes, specifically tested for the prevention of moderate and severe COPD exacerbations.
\end{abstract}

Keywords: hospitalization, COPD, triple therapy, inhaled corticosteroids, formoterol, glycopyrronium

\section{Introduction}

Acute exacerbations of COPD (AECOPDs) represent an acute event characterized by a rapid worsening of a patient's clinical state that requires a medical intervention of different intensity depending on the severity of the event and on the patient's basic condition. ${ }^{1}$ The real incidence of these events is difficult to measure, especially for the less severe cases that are certainly underestimated and underreported, being often documented in $<50 \%$ of cases. $^{2}$

The cost of AECOPDs represents the $50 \%$ of the direct economic burden of COPD; ${ }^{3}$ however, the cost increases up to $70 \%$ for exacerbations that require hospitalization ${ }^{3}-$ that is, severe exacerbations - and for the management of early recurrence, with a leading increasing cost of $18 \% .^{4}$

The prognosis of patients with COPD is influenced by the severity and recurrence of the exacerbation, with 1-year mortality rates ranging from $11 \%$ for patients who need hospitalization ${ }^{5}$ to $50 \%$ for patients subjected to mechanical ventilation. ${ }^{6}$ On the contrary, mortality increases up to $37 \%$ when hospitalization is needed in case of recurrent AECOPD. ${ }^{7}$ A prospective management strategy aimed at reducing
Correspondence: Francesco Blasi Department of Pathophysiology and Transplantation, University of Milan, Via Francesco Sforza 35, 20122 Milan, Italy Tel +390250320623

$\mathrm{Fax}+390250320625$

Email francesco.blasi@unimi.it 
the severity and the number of AECOPDs, with a particular focus on moderate and severe exacerbations, is needed in order to improve the patients' prognosis in terms of quality of life (QoL), disease progression and mortality, as well as the reduction in health care costs and the overall economic and social disease burden.

Nowadays, a consistent number of treatments have demonstrated this ability and are therefore suggested for the reduction of the rate and severity of acute exacerbations in patients with COPD. Nonetheless, to date, outcome-driven evidence coming from randomized clinical trials are limited, often leaving to the individual the clinical decision-making on the choice for the most appropriate treatment strategy to be used for patients with COPD at risk of exacerbation.

This narrative review explores the current evidence about the available pharmacological treatments for the prevention of AECOPDs, with a special focus on triple therapy and in particular on the combination of beclomethasone dipropionate (BDP)/formoterol (FORM)/glycopyrronium bromide (GLY).

\section{Role of inhaled corticosteroids (ICS) containing therapies in the prevention of exacerbations}

The indications for ICS containing therapies for patients with COPD have greatly changed during the last 15 years. Earlier, the widespread use of long-acting $\beta 2$-agonists (LABA) in combination with ICS was initially based upon the evidence of their efficacy in reducing AECOPD and hospitalizations. ${ }^{8,9}$ Subsequently, the UPLIFT trial demonstrated that also tiotropium (TIO), the first approved long-acting muscarinic antagonist (LAMA), was able to reduce the risk of AECOPD, making it a valid and safe alternative to the therapy with ICS/LABA. ${ }^{10,11}$

Previously, the growing evidence about efficacy and safety of LABA/LAMA fixed combinations and the increased risk of pneumonia linked with ICS-containing regimens further restricted the indication for ICS therapy, which was confirmed in patients at high risk of AECOPD that remained symptomatic or had severe airflow obstruction despite an initial trial with LABA/LAMA combinations.

The last release of the GOLD recommendations ${ }^{1}$ suggests that COPD patients at high risk of exacerbations - that is, patients with two or more AECOPD in the last 12 months - may benefit from the triple therapy, although the initial approach should always be based upon LABA/LAMA. Recently, expert opinion-based algorithms suggested that patients who would benefit more from ICS-containing therapies were patients with asthma-COPD overlap or with increased blood eosinophil counts. ${ }^{12,13}$

The indication toward the efficacy of ICS in preventing AECOPD, and especially severe exacerbations leading to hospitalization, has not always been inferred from randomized controlled trials (RCTs) that had as a primary outcome a reduction in the exacerbations rate. Moreover, throughout the years, the heterogeneity of patients enrolled and a nonunivocal definition of exacerbation determined contrasting results or data difficult to be interpreted in light of a real-life setting (Table 1).

In 2007, the results of the Towards a Revolution in COPD Health (TORCH) study were published. ${ }^{9}$ This was the first study that tested the twice daily (BID) fluticasone propionate/salmeterol (FP/SALM) 500/50 $\mu \mathrm{g}$ combination treatment in a consistent number of COPD patients. The primary outcome of the trial was mortality rate, which was not met, but at the end of the 3-year follow-up, patients in the combination treatment group had the lowest annual rate ratio of moderate and severe exacerbations (-25\% vs placebo). The annual rate ratio for the FP/SALM arm was also significantly lower compared with SALM or FP alone (risk ratio [RR] 0.88 and 0.91 , respectively). The advantage of the combination treatment over SALM alone, however, seemed to be no longer present when severe exacerbations were separately considered. Nonetheless, the trial proved that ICS-containing regimens, but not ICS alone, were efficacious in reducing hospitalization due to severe AECOPDs, improving QoL and lung function in patients with moderate to severe COPD.

In the same year, the Canadian Optimal Therapy of COPD Trial (OPTIMAL study) tested and compared the efficacy of FP/SALM 50/500 $\mu \mathrm{g}$ BID with TIO $18 \mu \mathrm{g}$ and the free association of TIO $18 \mu \mathrm{g}$ and SALM $50 \mu \mathrm{g}$ BID in reducing AECOPDs. ${ }^{23}$ The primary outcome was the proportion of patients in each arm who experienced an AECOPD during the 52 weeks of the trial. The trial found no difference in the AECOPD proportion between the two arms; however, the number of severe exacerbations leading to hospitalization was significantly reduced in the FP/SALM plus TIO arm compared with the other regimens - incidence rate ratio was 0.53 (0.33-0.86) compared with TIO alone. However, the trial was not powered to study the difference between the triple therapy regimen and the FP/SALM arm. Moreover, a dropout rate up to $40 \%$ in the TIO and TIO plus FP/SALM group made the study underpowered to meet the primary outcome.

Interestingly, the definition of COPD exacerbation for the TORCH and OPTIMAL trials was similar, described as 


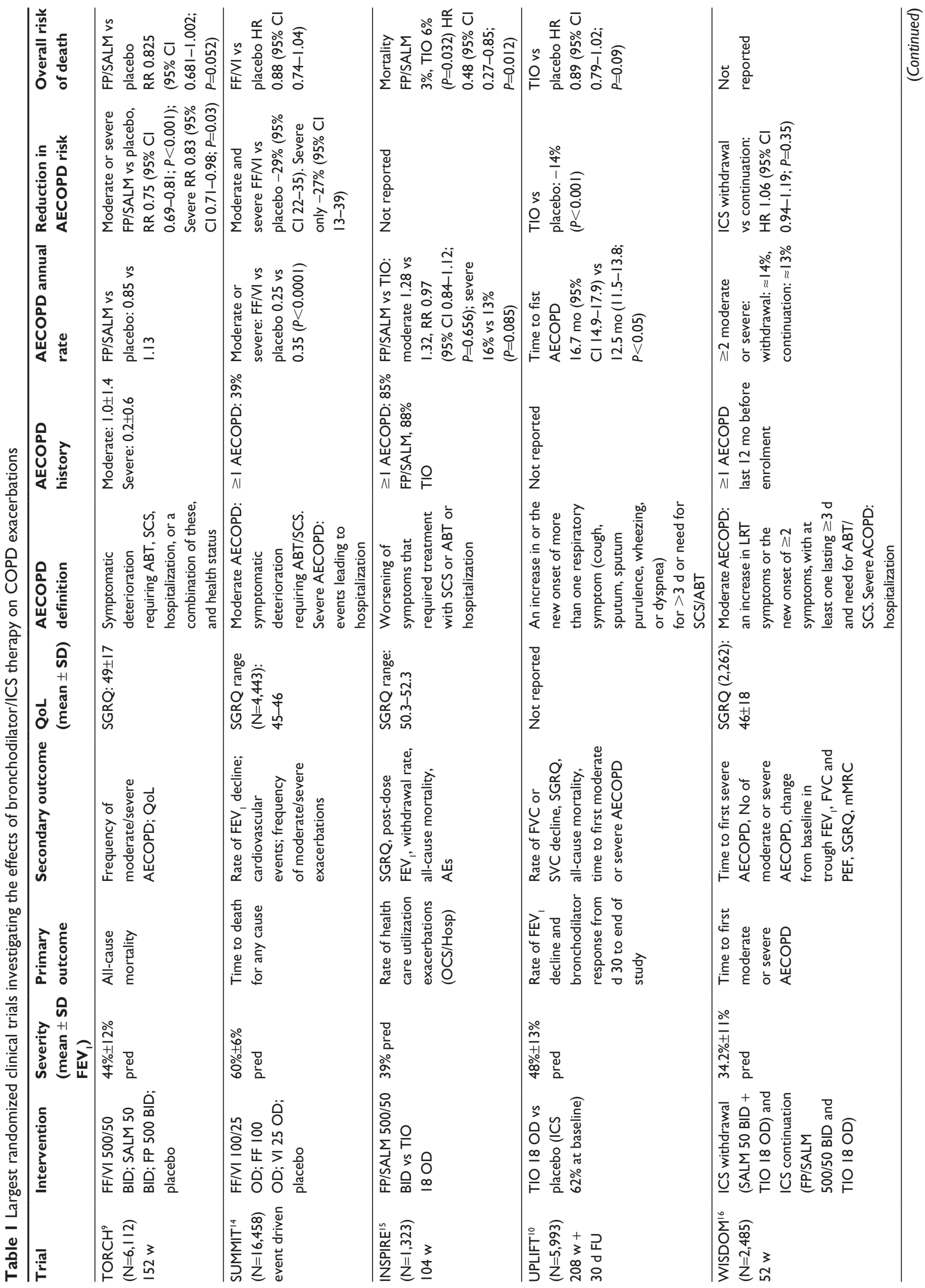




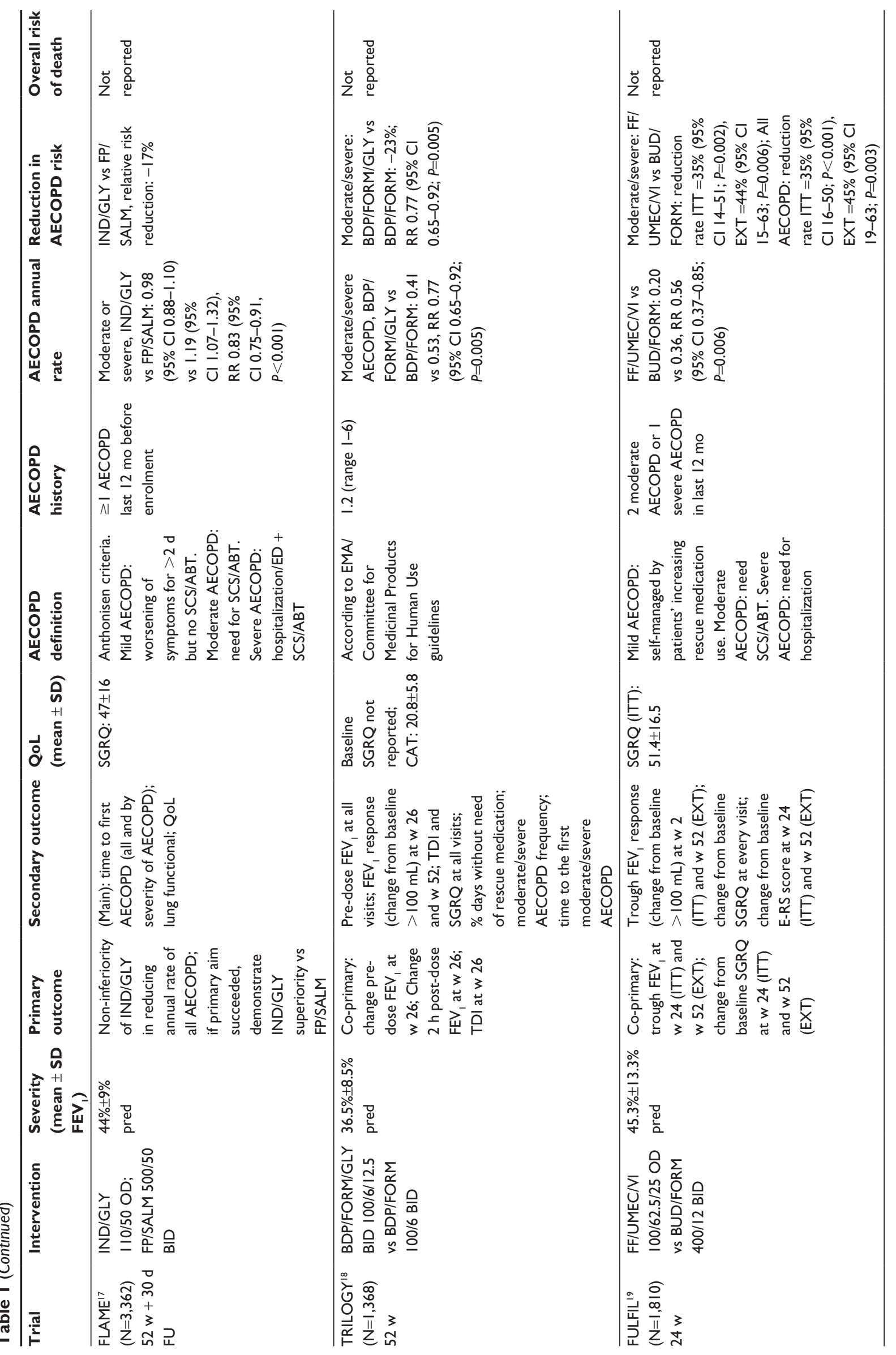


毞

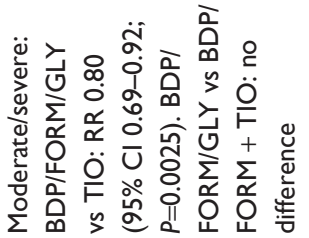

خ

$\sum_{\text {I }}$ O

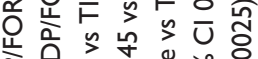

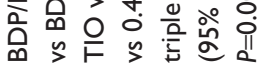

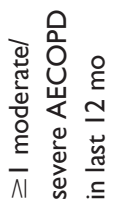

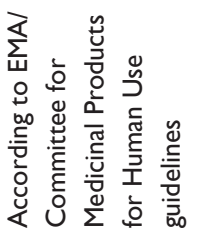

站
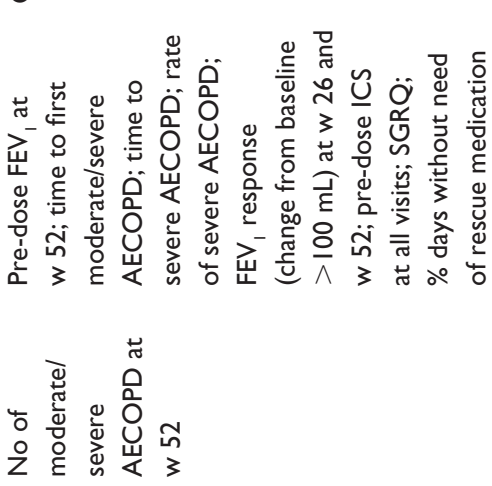

$\stackrel{\circ}{0}$
ò
+1
$\stackrel{0}{0}$
$\stackrel{m}{0}$

喜

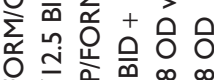

殅

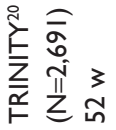

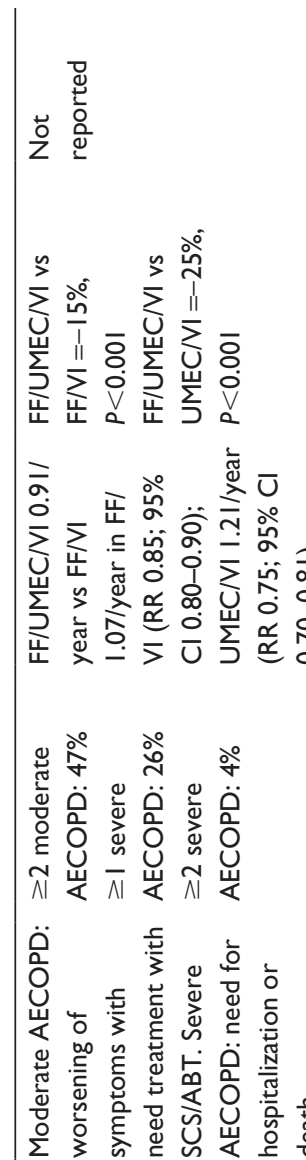

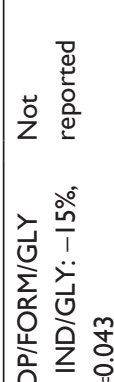

$\infty>$

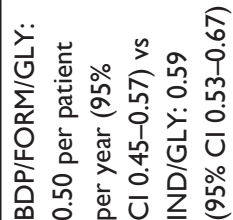

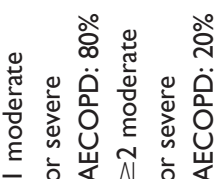

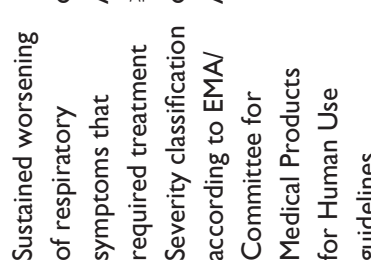

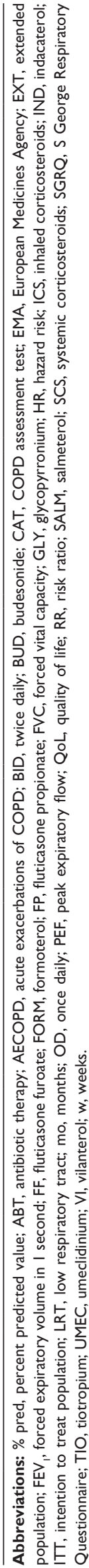


a deterioration of symptoms that required the use of systemic corticosteroids, antibiotics, or hospitalizations.

The INSPIRE was the first study designed to compare an ICS-containing regimen and the well-established efficacy of TIO in the exacerbation rate in severe patients with COPD. ${ }^{15}$ It was a 2-year multicenter, randomized double-blind, double dummy controlled trial aimed at comparing the efficacy of FP/SALM 500/50 $\mu \mathrm{g}$ BID and TIO $18 \mu \mathrm{g}$ on the rate of moderate and severe COPD exacerbations over a 2-year period. The authors found no difference between the two treatment regimens, when both moderate and severe AECOPD were grouped and considering AECOPD leading to hospitalization. Although the study was not powered to detect such differences, patients treated with the ICS-containing regimen demonstrated a reduced mortality rate and better $\mathrm{QoL}$ compared with TIO. ${ }^{15}$ The INSPIRE was the first study to use two parallel definitions of COPD exacerbation: the first was event-based, defined as the requirement of health care resources, while the second one was symptom-based, according to the 1987 Anthonisen criteria. ${ }^{15}$

Given the aforementioned results and based on the evidence that the association of an ICS/LABA and TIO may provide additional clinical benefits than either the treatments alone in the real-life scenario, patients with severe airflow obstruction (forced expiratory volume in 1 second $\left[\mathrm{FEV}_{1}\right]<50 \%$ ) were commonly treated with the combination of TIO and an ICS-containing regimen..$^{24,25}$ The CLIMB (Evaluation of Efficacy and Safety of Symbicort as add-on Treatment to Spiriva in Patients with Severe COPD) trial was designed to provide clinical efficacy and safety data about the triple therapy regimen. ${ }^{24,25}$ This was a 12-week double-blind, parallel group RCT in which, after a run-in period on TIO $18 \mu \mathrm{g}$, patients with severe COPD (postbronchodilator $\mathrm{FEV}_{1}<50 \%$ predicted) were randomized to receive also budesonide (BUD)/FORM 320/9 $\mu \mathrm{g}$ or a matching placebo. ${ }^{24,25}$ The primary outcome was functional and consisted of the change in pre-morning dose of $\mathrm{FEV}_{1}$ from baseline to week 12 . The study met the primary outcome, demonstrating the superiority of the triple regimen in improving lung function in terms of both airflow obstruction and air trapping. Among the secondary outcomes, severe exacerbations were analyzed, and the combination of BUD/FORM with TIO seemed superior to TIO alone, decreasing the rate of severe AECOPD by $62 \%{ }^{24,25}$ Time to first exacerbation and time to first hospitalization were also reduced for the study arm treated with the triple regimen. The CLIMB study was not powered to effectively detect clinical meaningful differences in exacerbation rates, and due to the limited study duration, the results obtained, although impacting, were not sufficient to confirm their long-term validity. Further and not confirming results came from a 6-month RCT that compared FP/SALM 500/50 $\mu \mathrm{g}$ BID added to TIO $18 \mu \mathrm{g}$ and TIO alone in COPD patients with moderate to severe airflow obstruction. ${ }^{26}$ Also in this case, the primary outcome was based on lung function, while the effect on exacerbations was only a secondary endpoint. While proving the superiority of the free association of FP/SALM and TIO in lung function outcomes, the study failed to demonstrate any difference between the two treatments in terms of exacerbation rate. Similarly, the GLISTEN trial, ${ }^{27}$ although of limited duration and not sufficiently powered for the detection of exacerbation rate difference between the study groups, showed that the free association of GLY $44 \mu \mathrm{g}$ and FP/SALM 500/50 $\mu \mathrm{g}$ BID was not different in terms of exacerbation rate compared with TIO and FP/SALM or FP/SALM alone.

It is important to note that none of the mentioned RCTs had, among their inclusion criteria, a consistent history of exacerbations. To enter the TORCH study, patients had to have at least one exacerbation in the previous 12 months, while for the INSPIRE trial, the positive history of AECOPD was not numerically specified. The study by Hanania et al, ${ }^{26}$ on the contrary, reported that on average only $24 \%$ of patients had at least one AECOPD in the 12 months before enrolment, making difficult to draw any clinical conclusion on exacerbation-related data.

Soon later, Short et al performed the first retrospective cohort analysis aimed at comparing the risk of exacerbation and mortality in patients treated with ICS/LABA plus TIO or ICS/LABA alone. ${ }^{28}$ The triple therapy regimen resulted to reduce the risk of exacerbation by $29 \%$ and the hospital admissions by $15 \%$ compared with ICS/LABA alone during an average follow-up of 4.65 years. ${ }^{28}$ The triple therapy also proved to reduce the all-cause mortality by $35 \%$ compared with ICS/LABA. ${ }^{28}$ The study was limited by its retrospective and observational design, but provided further indirect evidence that a combination of a LAMA with a ICS/LABA could be beneficial in terms of AECOPD prevention. ${ }^{28}$

The introduction of the first ultra-LABA indacaterol (IND) made it possible to test for the first time whether a combination of LAMA glycopyrronium with IND was able to reduce the risk of exacerbations in patients with severe COPD compared with GLY or TIO alone. ${ }^{29}$ The primary aim of the SPARK study ${ }^{29}$ was to demonstrate the superiority of the fixed dose association of IND and GLY in reducing the rate of moderate or severe AECOPD 
compared with GLY. The duration of the study was 64 weeks (that could be prolonged up to 76 weeks), and the study enrolled patients with a severe airflow obstruction and with at least one AECOPD in the previous 12 months. ${ }^{29}$ The LABA/LAMA treatment seemed to be superior in reducing moderate or severe exacerbations $(-12 \%)$, mild exacerbations $(-15 \%)$, and all exacerbations considered together $(-15 \%)$ compared with GLY alone. However, there was no statistical difference in the rate of severe exacerbations, both compared with TIO and GLY. ${ }^{29}$

In light of the equivalent effect on AECOPD demonstrated in the INSPIRE trial and the significant effect of the LAMA/LABA combination, although limited mainly on mild exacerbation when compared with TIO, the FLAME study was designed to offer a direct comparison between the LABA/LAMA IND/GLY once daily (OD) and FP/ SALM 500/50 $\mu \mathrm{g}$ BID in a 52-week RCT. ${ }^{17}$ Accordingly, the authors' primary objective was to demonstrate the non-inferiority of the double bronchodilator regimen compared with the ICS/LABA and that the primary outcome consisted of the annual rate of all AECOPD (including mild, moderate, and severe). ${ }^{17}$ Included patients had a FEV between $25 \%$ and $60 \%$ predicted and at least one exacerbation in the 12 months prior the enrolment in the study. ${ }^{17}$ A significant reduction in the rate of all AECOPDs was found in patients treated with IND/GLY group (intention to treat [ITT] population RR 0.89 (0.83-0.96), $P=0.003)$; this was also true for the time to first exacerbation, for which the LABA/LAMA regimen proved to be superior to $\mathrm{FP} / \mathrm{SALM}$ both in moderate or severe and in severe AECOPD considered separately. ${ }^{17}$ However, subgroup analyses, especially including patients with very severe airflow obstruction, symptomatic patients at low risk of exacerbations (GOLD group B), and patients with two or more exacerbations in the previous year (the so-called frequent exacerbators), showed a possible role of ICScontaining regimens. ${ }^{17}$

Studies published before the FLAME trail demonstrated a possible correlation between the peripheral eosinophil level and the response to ICS in AECOPD reduction. Contrary to what is expected, the FLAME trial demonstrated a similar effect of the two treatment regimens in patients with both $<2 \%$ and $>2 \%$ of serum eosinophils. ${ }^{17}$ The same was reported to be true for cutoff values other than $2 \%$ that were tested..$^{30}$ All considered, in the "pre-triple" fixed dose combination (FDC) era, the evidence of the efficacy of ICScontaining regimens in terms of reduction of AECOPD risk was mostly derived from data from the secondary outcomes and extrapolated from studies comparing an ICS/LABA with a LABA alone. Prospective RCT that investigated free combination therapies with a LAMA and an ICS/LABA did not provide definitive proofs of the superiority of the triple therapy compared with ICS/LABA FDC.

Main characteristics and results of the largest RCTs are summarized in Table 1.

\section{Focus on FORM, GLY, and BDP Pharmacokinetics and pharmacodynamics of FORM}

FORM (eformoterol in the UK) is a $\beta 2$-adrenoreceptor agonist approved by the European Medicine Agency and the Food and Drug Administration for the maintenance treatment of COPD and asthma. It is a phenylethanolamine derivative, a pure diastereomer of the R,R- and S,S-enantiomers of 2-hydroxy-5-[(1R,S)-1-hydroxy-2-][(1R,S)-2-(pmethoxyphenyl)-1-methylethylamino] formanilide prepared as the fumarate dihydrate salt. Its structural features allow a high selectivity, intrinsic activity, and very high affinity at the $\beta 2$-adrenoceptor. ${ }^{31}$ Relatively high water solubility and moderate lipophilicity ensure rapid access of inhaled FORM to the $\beta 2$-adrenoceptor on bronchial smooth muscle cells and rapid bronchodilation. ${ }^{31}$ In contrast to the LABA SALM, the uptake of FORM by airway smooth muscle cells is dependent on organic cation transporter $3 .^{32}$ The lipophilicity of FORM also explains its prolonged duration of action, ${ }^{33}$ and the possibility to be stored in cell membranes, making a depot from which the drug is available to $\beta 2$-adrenoceptors on bronchial smooth muscle cells. ${ }^{34}$ The reported pKi (negative logarithm of the equilibrium dissociation constant of a ligand determined in inhibition studies) for FORM are from $6.07 \pm 0.04$ to $6.12 \pm 0.09$ and from $7.84 \pm 0.05$ to $8.29 \pm 0.03$, for $\beta 1$ and $\beta 2$ receptors, respectively, while the intrinsic activity (\% isoprenaline) varies between 90 to 100 , depending on the reports. ${ }^{35}$ FORM also has the highest bronchoselectivity compared with $\beta 2$-adrenoceptor agonists isoproterenol, orciprenaline, trimetoquinol, and albuterol. ${ }^{36}$ The functional selectivity (ratio $\beta 1: \beta 2$ ) of FORM ranges from 1:13 to 1:166 and SALM from 1:525 to $1: 3,000 .{ }^{35}$

The bronchodilator effect of FORM in animal models was the most potent compared with other four $\beta 2$-adrenoceptor agonists (isoproterenol, orciprenaline, trimetoquinol, and albuterol), after either subcutaneous, oral, or aerosol administration. ${ }^{36,37}$ FORM's high affinity for $\beta 2$-adrenoceptors and high intrinsic efficacy are also responsible for doserelated inhibition of histamine-induced bronchoconstriction, being 10 - to 20 -fold more potent than either albuterol or 
SALM. ${ }^{36,38,39}$ In vitro and in vivo studies have shown that FORM has a fast onset of action that occurs within minutes after inhalation, ${ }^{38,40}$ comparable to that of albuterol and faster than SALM. ${ }^{39}$ Fast onset of action was described for FORM $12 \mu \mathrm{g}\left(\right.$ Modulite $\left.^{\circledR}\right)$ administered alone or in combination with TIO $18 \mu \mathrm{g}$ during mild and moderate COPD acute exacerbations. ${ }^{41}$ The duration of action of FORM, approved as a BID administration of LABA, is longer than that of albuterol but markedly shorter than that of SALM. ${ }^{42}$

\section{Pharmacokinetics and pharmacodynamics of GLY}

GLY, the active moiety of its bromide salt glycopyrrolate, is an antimuscarinic drug, first approved by the Food and Drug Administration (FDA) for the management of sialorrhea due to preoperative stages, neuromusclular and drug-related diseases, and gastric secretions. ${ }^{43,44}$ It is a quaternary ammonium derivative initially approved by FDA, European Medicines Agency, and in Japan as a monotherapy for COPD. ${ }^{45}$ In Europe, GLY was approved with a OD posology, whereas in the USA the BID regimen is recommended. Recently, FDA approved its BID posology in combination with fluticasone at a dosage of $9 \mu \mathrm{g}$ BID delivered with a pressurized metered-dose inhaler (pMDI) formulation. GLY is rapidly absorbed into the systemic circulation after inhalation, $\approx 90 \%$ via lung and $\approx 10 \%$ via gastrointestinal absorption. ${ }^{46}$

The mean terminal elimination half-life $\left(t_{1 / 2}\right)$ of GLY in dry powder inhaler formulations is half that reported for GLY delivered by pMDI (from 13 to 22 hours for a dose range of 25 to $200 \mu \mathrm{g}$ and from 6.3 to 9.6 hours for a dose range of 14.4 to $115.2 \mu \mathrm{g}$, respectively). ${ }^{47}$

GLY is a competitive muscarinic receptor antagonist that bronchodilates the airways by inhibiting acetylcholine-induced bronchoconstriction in bronchial smooth muscle cells. ${ }^{48}$

The three main muscarinic receptors related to human lung bronchial dynamics are the M1, M2, and M3 receptors. M2 receptor protects against bronchoconstriction and its block may increase heart rate. ${ }^{48,49} \mathrm{M} 1$ and, especially, M3 receptors are thought to be the prime mediators of the vagalinduced bronchoconstriction. ${ }^{46,48}$ In vitro, GLY has 4 - to 5-fold higher selectivity for human M1 and M3 receptors compared with M2 receptor. ${ }^{46,50}$ GLY has a faster onset of action in both in vitro and in vivo animal models compared with $\mathrm{TIO} .{ }^{50}$ The lower equilibrium binding affinity constants and the faster kinetic off rate justify the faster onset of action in patients with moderate to severe COPD compared with tiotropium bromide. ${ }^{51}$ In patients with severe COPD, GLY showed a fast and comparable ability to improve static lung volumes compared with aclidinium. ${ }^{52}$

GLY has been shown to be more potent than ipratropium bromide and tiotropium bromide in terms of concentration drug necessary to inhibit the contractile response by $50 \%{ }^{53}$

\section{Pharmacokinetics and pharmacodynamics of BDP}

BDP is the dipropionate ester of the free form beclomethasone-17-monopropionate, a synthetic halogenated glucocorticoid. BDP is a prodrug that must be activated through enzymatic processes in the lungs. ${ }^{45}$ Despite its higher oral availability compared with other ICSs, its prodrug form reduces the risk of local pharyngeal side effects, while it may favor a better pulmonary targeting, with $95 \%$ of beclomathasone that reaches the lung being transformed in its active form BDP delivered by pMDI has showed one of the highest lung deposition rates, reaching $>50 \%$ of the delivered dose. ${ }^{54,55}$

BDP has the lower known receptor affinity among mostly used ICSs. Given the binding ability of dexamethasone as 100, BDP has 53, while for comparison, salmeterol has a receptor affinity of 1,800 . Although a higher affinity may signify higher potency, and thus, the possibility to employ lower dosages to exert the desired effect, a higher binding ability, may promote adverse effects. ${ }^{56,57}$ Nonetheless, given different binding affinities, ICSs may exert similar pulmonary targeting and thus similar anti-inflammatory effects.

\section{Interaction and advantages of triple combination therapy}

The small airways are the major and earlier site of lung damage in COPD,${ }^{58}$ and their dysfunction is responsible for pathophysiological effects that withstand respiratory symptoms such as dyspnea, airway hyper-responsiveness, and exercise limitation. Both LABAs and LAMAs have demonstrated beneficial effects in terms of reduction in air trapping, closing volume, and airway stretching, ${ }^{59-61}$ especially when administered together. ${ }^{62}$ The rationale of combining a LABA and a LAMA relies on the synergistic effect exerted by the two classes of bronchodilators at the pre-junctional and intracellular levels. ${ }^{62}$ In fact, the stimulation of pre-junctional $\beta 2$-receptors both reduce the intracellular calcium concentration, thus reducing the release of acetylcholine, and stimulate adenylyl cyclase that indirectly allows the release of acetylcoline in the synaptic space. On the contrary, LAMAs inhibit M3 receptors and amplify the bronchodilation induced 
by LABAs. Moreover, more complex additive effects are elicited through the intracellular crosstalk between M3, M2, and $\beta 2$-receptors. However, low and iso-effective concentrations are needed in order to express a significant additive bronchodilation effect on the airways, the latter being more a class effect rather than being linked to a specific molecule. ${ }^{63}$ A dose-response trial that evaluated the most effective concentration of extra fine GLY added to extra fine BDP/ FORM 200/12 $\mu$ g FDC daily demonstrated that the dose of $25 \mu \mathrm{g}$ exerts the ideal dose-effect in bronchodilation when compared with BDP/FORM alone and GLY doses of 12.5 and $50 \mu \mathrm{g} .{ }^{64}$ In addition, the combination of FORM and BDP proved to have a strong synergistic effect at very low doses ( 6 and $100 \mu \mathrm{g}$, respectively) in small hyper-responsive human airways. Despite the great technological evolution during recent years, inhaled drug deposition is jeopardized by lung ventilation inhomogeneity, unpredictable regional airflow/obstruction characteristics, and the impacts of majority of inhaled drugs in the larger airways. The possibility of a synergistic effect at very low doses becomes crucial when considering the bronchodilator and anti-inflammatory effects exerted at the small airway level. In fact, the increased small particle fraction granted by the pMDI formulation of BDP/ FORM/GLY should promote a better peripheral drug deposition, thus allowing for clinical effectiveness despite the small absolute amount of active drug. ${ }^{65}$ Although currently no in vitro study has evaluated the iso-effectiveness and the proportionality of the GLY and FORM dosages in the triple FDC, the effects of the triple therapy observed in patients with recurrent exacerbations and a consistent small airway damage represent a first evidence of the balance and function and clinical effectiveness of this combination. While the synergistic effect of the combination of aclidinium and FORM or GLY and IND has been proven in in vitro and translational studies, this is not true for the combination of vilanterol (VI, under-dosed) and umeclidinium (UMEC, over-dosed) ${ }^{63,66}$

\section{Triple therapy for the management of moderate/severe exacerbations}

The TRILOGY, FULFIL, and TRINITY studies were the three RCTs that evaluated the efficacy of FDC triple therapy in COPD for the first time. ${ }^{18-20}$

TRIBUTE $^{22}$ and IMPACT ${ }^{21}$ were recently published and add new important information about this therapeutic approach to COPD treatment. The TRILOGY, ${ }^{18}$ TRINITY,${ }^{20}$ and TRIBUTE ${ }^{22}$ trials compared the efficacy of the FDC of BDP/FORM/GLY $100 / 6 / 12.5 \mu \mathrm{g}$ with the FDC of
BDP/FORM (TRILOGY), the free combination of BDP/ FORM with TIO or TIO alone (TRINITY), and the FDC of the LABA/LAMA IND/GLY (TRIBUTE). The FULFIL tria $1^{19}$ compared the efficacy of the FDC of fluticasone furoate/umeclidinium/vilanterol (FF/UMEC/VI) 100/62.5/25 $\mu \mathrm{g}$ with the FDC of BUD/FORM, while the IMPACT study ${ }^{21}$ compared the FF/UMEC/VI FDC with the LAMA/LABA FDC UMEC/VI and the ICS/LABA FDC FF/VI.

Trilogy was a randomized, parallel group, double-blind, active-controlled study performed in 159 sites across 14 countries that compared the efficacy of the combination of ICS/LABA LAMA, namely BDP/FORM/GLY in one inhaler with the fixed combination of BDP/FORM over 52 weeks. ${ }^{18}$

FULFIL trial was a Phase III, randomized, double-blind, double-dummy, parallel group, multicenter study that compared the efficacy of the combination in one inhaler of FF, UMEC, VI to BUD/FORM over 24 weeks. ${ }^{19}$ The study was designed to evaluate the efficacy in term of change of $\mathrm{FEV}_{1}$ from baseline at 24 weeks in all randomized subjects. An extension of the study up to 52 weeks was intended in a subgroup of patients for safety purposes. ${ }^{19}$

TRINITY was a randomized, parallel group, doubleblind, double-dummy, active-controlled 1-year trial, performed in 224 sites across 15 countries that compared the efficacy of three different treatments: BDP/FORM/GLY FDC; TIO monotherapy; and the combination therapy with BDP/FORM added to TIO. ${ }^{20}$

IMPACT was a randomized, double-blind, parallel-group, multicenter trial on Phase III that compared the efficacy over 52 weeks of OD combination of FF $(100 \mu \mathrm{g})$, UMEC at the dose of $62.5 \mu \mathrm{g}$ and VI $(25 \mu \mathrm{g})$ on the combination of FF/VI or the combination of UMEC/VI. ${ }^{21}$

TRIBUTE was a randomized, parallel group, doubleblind, double-dummy, active-controlled Phase IIIb study that compared the effect of one inhaler combination of two bronchodilator, namely IND $85 \mu \mathrm{g} / \mathrm{GLY} 43 \mu \mathrm{g}$ OD and one inhaler triple therapy with BDP/FORM/GLY nominal dose respectively of $100 \mu \mathrm{g} / 6 \mu \mathrm{g} / 9 \mu \mathrm{g}$ BID. ${ }^{22}$

The studies explored different aspects of the disease; TRILOGY and FULFILL set lung function and QoL as the primary outcomes and evaluated AECOPDs as secondary outcomes while TRINITY, IMPACT, and TRIBUTE evaluated moderate and severe AECOPDs as the main outcomes (Table 1).

TRILOGY study randomized 1,368 patients while 1,810 patients were included in the ITT population of the FULFIL study and 430 were included in the extension (EXT) population. Both the studies demonstrated that adding a LAMA to 
different combinations of ICS/LABA improved lung function and QoL, but not dyspnea. ${ }^{18,19}$

In the TRILOGY study, the percentage of patients who had moderate-to-severe AECOPD was lower with BDP/ FORM/GLY (31\%) than with BDP/FORM (35\%). The adjusted annual rate of moderate-to-severe exacerbations was 0.41 for the BDP/FORM/GLY group and 0.53 for the BDP/FORM group, with a rate ratio of 0.77 (95\% CI $0.65-0.92 ; P=0.005)$, indicating a significant $23 \%$ reduction in the exacerbation rate with BDP/FORM/GLY. Subgroup analyses of patients with more than one AECOPD have shown a more consistent treatment effect, with a reduction rate of moderate/severe AECOPD of 33\% in patients treated with BDP/FORM/GLY compared with the BDP/FORM group (rate ratio 0.67 [95\% CI 0.48-0.94]; $P=0.019$ ), whereas a minor effect could be found in the subgroup of patients with a history of one exacerbation or less in the previous 12 months (RR 0.83 [95\% CI 0.67-1.02]; $P=0.074) .^{18}$

Different from TRINITY and TRILOGY trials, the FULFIL inclusion criteria were based not solely on exacerbations history, but included patients who belonged to group D according to GOLD classification 2016, that is, either patients with a $\mathrm{FEV}_{1}<50 \%$ predicted and increased symptoms or patients with $\geq 1$ severe exacerbation or $\geq 2 \mathrm{mild} /$ moderate AECOPD. In the ITT population, $65 \%$ of patients had at least one moderate/severe exacerbation. The incidence rates of moderate/severe AECOPD over the 24-week treatment period were $10 \%$ and $14 \%$ for FF/UMEC/VI and BUD/ FORM, respectively. The mean annualized rates of moderate/ severe exacerbations in the ITT population were 0.22 and 0.34 for FF/UMEC/VI and BUD/FORM, respectively, while the reduction in the annualized rate was statistically significant for FF/UMEC/VI (35\%; 95\% CI 14\%-51\%; $P=0.002) .{ }^{19}$ Patients treated with FF/UMEC/VI experienced AECOPDs less frequently leading to hospitalization $(n=12$, $1 \%$ ) compared with the BUD/FORM treatment group $(n=22,2 \%){ }^{19}$

AECOPDs were the focus in the TRINITY, IMPACT, and TRIBUTE studies that were designed to evaluate the rate of moderate/severe AECOPDs as the primary endpoint. In the TRINITY study, the comparison was made between the triple therapy and the LAMA alone and not compared with ICS/LABA. ${ }^{20}$

The results show that in the FDC triple therapy (BDP/ FORM/GLY) group, the exacerbation rates were $0.46(0.41-$ $0.51)$ per patient, while in the TIO arm were $0.57(0.52-0.63)$ with an RR of $0.80(95 \%$ CI $0.69-0.92 ; P=0.0025)$. In the
BDP/FORM plus TIO in open, the exacerbation rate was $0.45(0.39-0.52)$ with a lower RR compared with patients treated with TIO (RR 0.79; 95\% CI 0.66-0.94; $P=0.0095$ ). ${ }^{20}$ No difference was found with the FDC triple therapy (RR $1.01 ; 95 \%$ CI $0.85-1.21 ; P=0.89) .{ }^{20}$

The TRINITY study explored the effect of the inhaled treatment on exacerbation also in terms of the time to first moderate-to-severe exacerbation as a secondary endpoint. ${ }^{20}$ The results have shown that FDC triple therapy was able to prolong the time to the next exacerbation compared with TIO alone with a hazard ratio (HR) of 0.84 (95\% CI 0.72-0.97; $P=0.0154) .{ }^{20}$ However, the effect was similar compared with the group treated with BDP/FORM plus TIO in open (HR 1.06 ; $95 \%$ CI $0.88-1.27 ; P=0.57) .^{20}$

TRIBUTE was a randomized, parallel group, double-blind, double-dummy, active-controlled Phase IIIb trial that compared the efficacy of the FDC of BDP/FORM/GLY $100 / 6 / 12.5 \mu \mathrm{g}$ two actuations BID with the LABA/LAMA FDC IND/GLY $85 / 43 \mu \mathrm{g}$ OD. ${ }^{22}$ In the TRIBUTE trial, $87 \%$ of the 764 patients assigned to the BDP/FORM/GLY treatment group and $84 \%$ of the 768 patients assigned to IND/ GLY completed the study, with an adjusted rate ratio of moderate/severe AECOPD of 0.50 per patient/per year $(95 \%$ CI 0.45-0.57) and 0.59 (95\% CI 0.53-0.67), respectively. ${ }^{22}$ After 52 weeks of treatment, the FDC triple therapy showed a $15 \%$ reduction in moderate and severe exacerbation rate compared with the LABA/LAMA FDC, with an adjusted rate ratio of 0.85 (95\% CI $0.72-0.99 ; P=0.043) .{ }^{22}$ No significant differences in the exacerbation rate were found between the two treatment groups when moderate and severe exacerbations were analyzed separately. The annual exacerbation rate was significantly reduced by the FDC triple therapy in patient with $\geq 2 \%$ of peripheral eosinophils (RR 0.806 ; $95 \%$ CI $0.664-0.978 ; P=0.029)$, and the trend was maintained considering the absolute cutoff of 200 eosinophils/ $\mu \mathrm{L}$ (RR 0.806; 95\% CI 0.646-1.007; $P=0.057) .{ }^{22}$ An increase rate of pneumonia was not present in patients treated with BDP/ FORM/GLY, while atrial fibrillation was more frequently observed in the IND/GLY group. ${ }^{22}$

The IMPACT was a Phase III, randomized, double-blind, parallel-group, multicenter trial. The primary outcome was the effect of the FDC of FF/UMEC/VI 100/62.5/25 $\mu \mathrm{g}$ OD compared with the LABA/LAMA combination of UMEC/ VI $100 / 25 \mu \mathrm{g}$ OD and the ICS/LABA FDC of FF/VI $100 / 25 \mu \mathrm{g}$ OD in terms of moderate or severe AECOPD rate after 52 weeks of treatment. Patients were enrolled if they had a COPD assessment test (CAT) score $\geq 10$ and either a $\mathrm{FEV}_{1}<50 \%$ predicted and a history of at least 


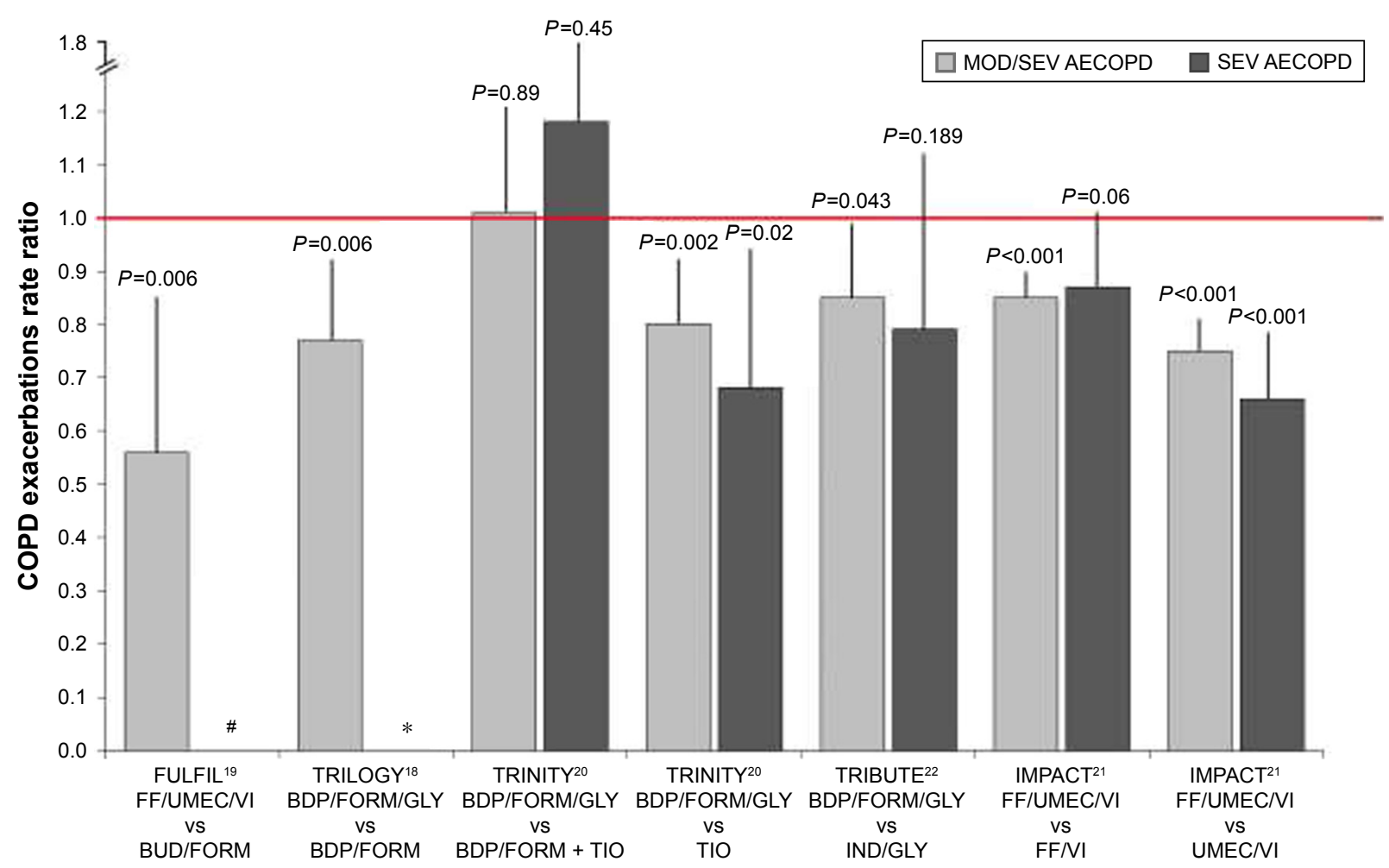

Figure I Relative rate ratio for moderate/severe and severe COPD exacerbations in trials investigating efficacy of fixed-dose triple therapy compared with LABA/ICS, LAMA, LABA/LAMA, or open triple therapy.

Notes: Vertical bars represent the upper limit of the $\mathrm{Cl}$. The red line represents a $\mathrm{Cl}=\mathrm{I} .0$ and therefore the significance level. "Rate of severe exacerbations not reported; *Relative risk for severe exacerbations not available.

Abbreviations: MOD/SEV, moderate/severe exacerbations; SEV, severe exacerbations; LABA, long-acting $\beta 2$-agonists; ICS, inhaled corticosteroids; LAMA, long-acting muscarinic antagonist; AECOPD, acute exacerbations of COPD; FF, fluticasone furoate; BDP, beclomethasone dipropionate; FORM, formoterol; BUD, budesonide; GLY, glycopyrronium; TIO, tiotropium; IND, indacaterol; UMEC, umeclidinium; VI, vilanterol.

one moderate or severe exacerbation in the previous year, or a $\mathrm{FEV}_{1}$ between $50 \%$ and $80 \%$ predicted and at least two moderate exacerbations or one severe exacerbation in the previous year. ${ }^{21}$ At the end of the study period, the rate of moderate or severe exacerbations in triple therapy group was 0.91 per year compared with an annual rate of 1.07 for the FF/VI group (RR 0.85; 95\% CI 0.80-0.90; $P<0.001$ ) and 1.21 in the UMEC/VI group (RR $0.75 ; 95 \%$ CI $0.70-0.81$ ), suggesting a significant reduction in the rate of moderate and severe AECOPDs of $15 \%$ and $25 \%$, respectively. ${ }^{21}$ Considering severe AECOPDs only, the latter were low and the annual rate did not significantly differ $(0.13$ vs 0.15$)$ between the triple therapy groups compared with the group treated with ICS/LABA (RR $0.87 ; 95 \%$ CI 0.76-1.01; $P=0.06$ ). However, a statistically significant $35 \%$ reduction $(P<0.001)$ in severe exacerbations was reached comparing the effect of triple FDC with the LAMA/LABA UMEC/VI (RR 0.66; 95\% CI $0.56-0.78)$. Concurrently, patients with $>150$ eosinophil/ $\mu \mathrm{L}$ showed a not significant trend in reduction of moderate/ severe exacerbations when treated with triple therapy in FDC $^{21}$ (Figure 1).
The annual rate of pneumonia was significantly increased in patient treated with FF-containing regimens. The time to the first pneumonia event in patients treated with the FDC triple therapy and in the UMEC/VI group differed significantly (RR 1.53; 95\% CI 1.22-1.92; $P<0.001$ ), while no difference was found between the ICS-containing regimens. ${ }^{21}$ One of the main limitation of the study was the inclusion of a significant number of patients with an asthmatic trait, since $18 \%$ of the study sample had a significant reversibility defined as an increase of $12 \%$ and $200 \mathrm{~mL}$ from baseline after an acute bronchodilation test. ${ }^{67}$

\section{Perspective}

Making a research on ClinicalTrials.gov using the keywords "Chronic Obstructive Pulmonary Disease" and "Exacerbation" with the filter "Recruiting" and "not yet Recruiting" studies, by May 2018, we found 123 studies; of these, 11 evaluate the impact of inhaled treatments on AECOPDs and four are focused on triple therapy.

The SOPHOS study (NCT02727660) ${ }^{68}$ is a Phase III randomized, double-blind, parallel group, multicenter, 1-year 
trial completed in April 2018. It evaluates the effect of different doses of BUD/FORM delivered via pMDI compared with FORM alone on the reduction of mild, moderate, and severe exacerbations in COPD patients with severe airflow obstruction. At the moment, no study results are have been made available. ${ }^{68}$

TRIVERSYTI (NCT03197818) is an ongoing 24-week, double-blind, double-dummy, randomized, multinational, multicenter, two-arm parallel group, actively controlled clinical trial that compares the efficacy of the FDC BDP/ FORM/GLY delivered via pMDI with the FDC BUD/FORM in terms of lung function and time to first exacerbation in patients with severe COPD. ${ }^{69}$

Finally, ETHOS (NCT02465567) ${ }^{70}$ and AIRWISE $(\mathrm{NCT} 03265145)^{71}$ compare the effect of adding an ICS to a LABA/LAMA FDC treatment in the reduction of moderate/ severe AECOPD in severe COPD patients. The ETHOS study tests different doses of the FDC of BUD/FORM/GLY with FF/GLY delivered via pMDI, ${ }^{70}$ while the AIRWISE study compares the TIO/olodaterol FDC with an ICS/LABA + LAMA regimen. ${ }^{71}$ The first study is planned to be competed by December 2018 and the second by January 2020 .

\section{Pharmacological prevention of moderate and severe COPD exacerbations}

The triple therapy approach is one of the proposed options for the management of patients with frequent exacerbations. Although the initial strategy should be based upon treatment with a LABA/LAMA combination that had proved efficient in the reduction of mild and moderate AECOPDs, a definitive evidence on severe AECOPDs is still lacking. The evidence from trials investigating triple therapy regimens explored the effect of adding a second bronchodilator to an ICS/LABA regimen and the effect of adding an ICS to a LABA/LAMA treatment. Taken together, the results of the RCTs published so far show that there are good evidence that triple FDC combination is superior to TIO alone in the prevention of moderate to severe AECOPD. The evidence on the prevention of AECOPDs from the TRILOGY ${ }^{18}$ and FULFIL ${ }^{19}$ trials seem to suggest that FDC triple therapy could reduce moderate and severe exacerbations more effectively than an ICS/LABA combination therapy. The effect seems to be more pronounced in patients with more than one moderate/ severe AECOPD in the year preceding the enrolment. The IMPACT and TRIBUTE trials were specifically designed to explore the effect of a triple FDC therapy in the reduction of moderate and severe exacerbations in patients with a severe airflow obstruction and with a significant history of exacerbations. ${ }^{21,22}$ Data from these two large trials indicate a positive effect of the triple combination therapy in terms of reduction in moderate/severe exacerbations taken as a whole. However, when comparing the triple FDC with LABA/LAMA in the reduction of severe exacerbations, data are discordant. In fact, while patients treated with the FF/UMEC/VI FDC were less exposed to severe AECOPDs when compared with patients treated with the UMEC/VI regimen, the same was not apparent when comparing the effects of BDP/FORM/GLY and IND/GLY FDCs. The percentage of frequent exacerbators (patients with $\geq 2$ exacerbations in the previous year) in the IMPACT trial was around $47 \%$ in each treatment arm, ${ }^{21}$ while the latter was low as $20 \%$ and $18 \%$ in the BDP/FORM/GLY and IND/GLY arms in the TRIBUTE trial. This large difference in the characteristics of the study populations between the two trials may have hindered the possible differences between the ICS/LABA/ LAMA and LABA/LAMA in terms of reduction in severe exacerbations in the TRIBUTE study. ${ }^{22}$

Confirming previous observations, ${ }^{17,30}$ the effect of the triple therapy in the IMPACT trial seemed to be independent of the peripheral eosinophil level. On the contrary, results from the TRIBUTE trial suggest that patients with $\geq 2 \%$ of serum eosinophils could respond better to an FDC triple therapy in the prevention of exacerbations. Recent evidence from the SUNSET trial ${ }^{72}$ shows that stepping down from triple therapy to a LAMA/LABA combination in non-frequent exacerbators can be safe in patients with an eosinophil count $\leq 300$ cells $/ \mu \mathrm{L}$, while patients with higher eosinophil concentration may experience a larger decrease in lung function and a slightly higher risk of future exacerbation. Based on these results, eosinophils may still have a role in the decisionmaking for long-term COPD treatment, but, as also previously stated by Calverley, ${ }^{73}$ the most important and consistent parameter seems to be the history of moderate and severe exacerbations in patients already treated with a LABA/LAMA therapy. No less importantly, data from the IMPACT study confirm the possible increased risk of pneumonia in patients treated with fluticasone-containing inhaled regimens.

\section{Author contributions}

All authors contributed toward data analysis, drafting and revising the paper and agree to be accountable for all aspects of the work.

\section{Disclosure}

MM has received reimbursement for travel and conference participation by Boehringer Ingelheim, Menarini, AstraZeneca and payment for teaching in training course by Guidotti, Malesci, 
and Grifols. DR has received speaker honoraria from Boehringer Ingelheim and AstraZeneca. PS received grants and personal fees from Chiesi Farmaceutici and Boehringer Ingelheim; grants from Air Liquide, Pfizer, and Almirall; and personal fees from AstraZeneca, GSK, Novartis, Menarini, Malesci, Guidotti, Mundipharma, and Zambon. FB received speaker or consultant honoraria or research funding from Menarini, Almirall, AstraZeneca, Boehringer Ingelheim, Chiesi Farmaceutici, Dompe, GlaxoSmithKline, Lab. Guidotti, Malesci, Mundipharma, Novartis, Pfizer, Teva, Valeas, and Zambon. The authors report no other conflicts of interest in this work.

\section{References}

1. goldcopd.org. [homepage on the Internet]. From the Global Strategy for the Diagnosis, Management and Prevention of COPD, Global Initiative for Chronic Obstructive Lung Disease (GOLD); 2017 [updated 2017; cited Jan 18, 2018]. Available from: http://goldcopd.org. Accessed January 18, 2018.

2. Seemungal TA, Donaldson GC, Bhowmik A, Jeffries DJ, Wedzicha JA. Time course and recovery of exacerbations in patients with chronic obstructive pulmonary disease. Am J Respir Crit Care Med. 2000;161: 1608-1613.

3. Strassels SA, Smith DH, Sullivan SD, Mahajan PS. The cost of treating COPD in the United States. Chest. 2001;119:344-352.

4. FitzGerald JM, Haddon JM, Bradly-Kennedy C, Kuramoto L, Ford GT; RUSIC Study Group. Resource use study in COPD (RUSIC): a prospective study to quantify the effects of COPD exacerbations on health care resource use among COPD patients. Can Respir J. 2007;14:145-152.

5. Miguel-Diez J, Jimenez-Garcia R, Hernandez-Barrera V, et al. Trends in hospital admissions for acute exacerbation of COPD in Spain from 2006 to 2010. Respir Med. 2013;107:717-723.

6. Halpin DMG, Decramer M, Celli B, Diette GB. Exacerbation frequency and course of COPD. Int J Chron Obstruct Pulmon Dis. 2012;7:653-661.

7. Patil SP, Krishnan JA, Lechtzin N, et al. In-hospital mortality following acute exacerbations of chronic obstructive pulmonary disease. Arch Intern Med. 2003;163:1180-1186.

8. Calverley PM, Boonsawat W, Cseke Z, Zhong N, Peterson S, Olsson H. Maintenance therapy with budesonide and formoterol in chronic obstructive pulmonary disease. Eur Respir J. 2003;22:912-919. Erratum in: Eur Respir J. 2004;24:1075.

9. Calverley PM, Anderson JA, Celli B, et al; TORCH investigators. Salmeterol and fluticasone propionate and survival in chronic obstructive pulmonary disease. $N$ Engl $J$ Med. 2007;356:775-789.

10. Tashkin DP, Celli B, Senn S, et al; UPLIFT Study Investigators. A 4-year trial of tiotropium in chronic obstructive pulmonary disease. N Engl J Med. 2008;359:1543-1554.

11. Santus P, Di Marco F, Radovanovic D, Centanni S. Tiotropium: what came after the UPLIFT study. Expert Opin Pharmacother. 2012; 13:613-618.

12. Miravitlles M, Anzueto A. A new two-step algorithm for the treatment of COPD. Eur Respir J. 2017;49:1600791.

13. Cooper CB, Barjaktarevic I. A new algorithm for the management of COPD. Lancet Respir Med. 2015;3:266-268.

14. Vestbo J, Anderson JA, Brook RD, et al; SUMMIT Investigators. Fluticasone furoate and vilanterol and survival in chronic obstructive pulmonary disease with heightened cardiovascular risk (SUMMIT): a double-blind randomised controlled trial. Lancet. 2016;387: $1817-1826$.

15. Wedzicha JA, Calverley PM, Seemungal TA, Hagan G, Ansari Z, Stockley RA; INSPIRE Investigators. The prevention of chronic obstructive pulmonary disease exacerbations by salmeterol/fluticasone propionate or tiotropium bromide. Am J Respir Crit Care Med. 2008; 177:19-26.
16. Magnussen H, Disse B, Rodriguez-Roisin R, et al; WISDOM Investigators. Withdrawal of inhaled glucocorticoids and exacerbations of COPD. N Engl J Med. 2014;371:1285-1294.

17. Wedzicha JA, Banerji D, Chapman KR, et al; FLAME Investigators. Indacaterol-glycopyrronium versus salmeterol-fluticasone for COPD. N Engl J Med. 2016;374:2222-2234.

18. Singh D, Papi A, Corradi M, Pavlisova I, Montagna I, Francisco C. Single inhaler triple therapy versus inhaled corticosteroid plus long acting $\beta$-agonist therapy for chronic obstructive pulmonary disease (TRILOGY): a double-blind, parallel group, randomized controlled trial. Lancet. 2016;388:963-973.

19. Lipson DA, Barnacle H, Birk R, et al. FULFIL trial: once-daily triple therapy for patients with chronic pulmonary disease. Am J Respir Crit Care Med. 2017;196(4):438-446.

20. Vestbo J, Papi A, Corradi M, et al. Single inhaler extrafine triple therapy versus long-acting muscarinic antagonist therapy for chronic obstructive pulmonary disease (TRINITY): a double-blind, parallel group, randomised controlled trial. Lancet. 2017;389:1919-1929.

21. Lipson DA, Barnhart F, Brealey N, et al; IMPACT Investigators. Oncedaily single-inhaler triple versus dual therapy in patients with COPD. N Engl J Med. 2018;378:1671-1680.

22. Papi A, Vestbo J, Fabbri L, et al. Extrafine inhaled triple therapy versus dual bronchodilator therapy in chronic obstructive pulmonary disease (TRIBUTE): a double-blind, parallel group, randomised controlled trial. Lancet. 2018;391:1076-1084.

23. Aaron SD, Vandemheen KL, Fergusson D, et al; Canadian Thoracic Society/Canadian Respiratory Clinical Research Consortium. Tiotropium in combination with placebo, salmeterol, or fluticasone-salmeterol for treatment of chronic obstructive pulmonary disease: a randomized trial. Ann Intern Med. 2007;146:545-555.

24. Cazzola M, Andò F, Santus P, et al. A pilot study to assess the effects of combining fluticasone propionate/salmeterol and tiotropium on the airflow obstruction of patients with severe-to-very severe COPD. Pulm Pharmacol Ther. 2007;20:556-561.

25. Singh D, Brooks J, Hagan G, Cahn A, O'Connor BJ. Superiority of "triple" therapy with salmeterol/fluticasone propionate and tiotropium bromide versus individual components in moderate to severe COPD. Thorax. 2008;63:592-598.

26. Hanania NA, Crater GD, Morris AN, Emmett AH, O'Dell DM, Niewoehner DE. Benefits of adding fluticasone propionate/salmeterol to tiotropium in moderate to severe COPD. Respir Med. 2012;106: 91-101.

27. Frith PA, Thompson PJ, Ratnavadivel R, et al; Glisten Study Group. Glycopyrronium once-daily significantly improves lung function and health status when combined with salmeterol/fluticasone in patients with COPD: the GLISTEN study, a randomised controlled trial. Thorax. 2015;70:519-527.

28. Short PM, Williamson PA, Elder DHJ, Lipworth SIW, Schembri S, Lipworth BJ. The impact of tiotropium on mortality and exacerbations when added to inhaled corticosteroids and long-acting $\beta$-agonist therapy in COPD. Chest. 2012;141:81-86.

29. Wedzicha JA, Decramer M, Ficker JH, et al. Analysis of chronic obstructive pulmonary disease exacerbations with the dual bronchodilator QVA149 compared with glycopyrronium and tiotropium (SPARK): a randomised, double-blind, parallel-group study. Lancet Respir Med. 2013;1:199-209.

30. Roche N, Chapman KR, Vogelmeier CF, et al. Blood eosinophils and response to maintenance chronic obstructive pulmonary disease treatment. Data from the FLAME trial. Am J Respir Crit Care Med. 2017;195: 1189-1197.

31. Anderson GP. Formoterol: pharmacology, molecular basis of agonism, and mechanism of long duration of a highly potent and selective beta 2-adrenoceptor agonist bronchodilator. Life Sci. 1993;52: 2145-2160.

32. Santus $\mathrm{P}$, Radovanovic D, Paggiaro $\mathrm{P}$, et al. Why use long acting bronchodilators in chronic obstructive lung diseases? An extensive review on formoterol and salmeterol. Eur J Intern Med. 2015;26: $379-384$. 
33. Anderson GP, Lindén A, Rabe KF. Why are long-acting beta-adrenoceptor agonists long-acting? Eur Respir J. 1994;7:569-578.

34. Lötvall J, Palmqvist M, Arvidsson P, Maloney A, Ventresca GP, Ward J. The therapeutic ratio of R-albuterol is comparable with that of RS-albuterol in asthmatic patients. J Allergy Clin Immunol. 2001;108:726-731.

35. Cazzola M, Page CP, Rogliani P, Matera MG. $\beta 2$ agonists therapy in lung disease. Am J Resp Care Med. 2013;187:690-696.

36. Cazzola M, Page CP, Calzetta L, Matera MG. Pharmacology and therapeutics of bronchodilators. Pharmacol Rev. 2012;64:450-504.

37. Ida H. Cardiorespiratory activities of 3-formylamino-4-hydroxy (N-1-methyl-2-p methoxyphenethylaminomethyl)-benzylalcoholhemifumarate (BD 40A) and some other -adrenoceptor stimulants in conscious guinea pigs. Arzneimittelforschung. 1976;26:1337-1340.

38. Lindén A, Bergendal A, Ullman A, Skoogh BE, Löfdahl CG. Salmeterol, formoterol, and salbutamol in the isolated guinea pig trachea: differences in maximum relaxant effect and potency but not in functional antagonism. Thorax. 1993;48:547-553.

39. Naline E, Zhang Y, Qian Y, et al. Relaxant effects and durations of action of formoterol and salmeterol on the isolated human bronchus. Eur Respir J. 1994;7:914-920.

40. Cazzola M, Centanni S, Regorda C, et al. Onset of action of single doses of formoterol administered via Turbuhaler in patients with stable COPD. Pulm Pharmacol Ther. 2001;14:41-45.

41. Di Marco F, Verga M, Santus P, Morelli N, Cazzola M, Centanni S. Effect of formoterol, tiotropium, and their combination in patients with acute exacerbation of chronic obstructive pulmonary disease: a pilot study. Respir Med. 2006;100:1925-1932.

42. Nials AT, Ball DI, Butchers PR, et al. Formoterol on airway smooth muscle and human lung mast cells: a comparison with salbutamol and salmeterol. Eur J Pharmacol. 1994;251:127-135.

43. Banfi P, Ticozzi N, Lax A, Guidugli GA, Nicolini A, Silani V. A review of options for treating sialorrhea in amyotrophic lateral sclerosis. Respir Care. 2015;60:446-454.

44. Blissit KT, Tillery E, Latham C, Pacheco-Perez J. Glycopyrrolate for treatment of clozapine-induced sialorrhea in adults. Am J Health Syst Pharm. 2014;71:1282-1287.

45. pubchem.ncbi.nlm.nih.gov [homepage on the Internet]. Pub Chem, Open Chemistry Database. National Center for Biotechnology Information; U.S. National Library of Medicine; 2005 [updated May 19, 2018]. Available from: https://pubchem.ncbi.nlm.nih.gov/compound/ glycopyrrolate. Last accessed May 15, 2018.

46. Carter NJ. Inhaled glycopyrronium bromide: a review of its use in patients with moderate to severe chronic obstructive pulmonary disease. Drugs. 2013;73:741-753.

47. Sechaud R, Renard D, Zhang-Auberson L, Motte Sde L, Drollmann A, Kaiser G. Pharmacokinetics of multiple inhaled NVA237 doses in patients with chronic obstructive pulmonary disease (COPD). Int $J$ Clin Pharmacol Ther. 2012;50:118-128.

48. Ulrik CS. Once-daily glycopyrronium bromide, a long-acting muscarinic antagonist, for chronic obstructive pulmonary disease: a systematic review of clinical benefit. Int J Chron Obstruct Pulmon Dis. 2012;7: 673-678.

49. Buhl R, Banerji D. Profile of glycopyrronium for once-daily treatment of moderate-to-severe COPD. Int J Chron Obstruct Pulmon Dis. 2012; 7:729-734.

50. Sykes DA, Dowling MR, Leighton-Davies J, et al. The influence of receptor kinetics on the onset and duration of action and the therapeutic index of NVA237 and tiotropium. J Pharmacol Exp Ther. 2012;343: 520-528.

51. Verkindre C, Fukuchi Y, Flémale A, et al. Sustained 24-h efficacy of NVA237, a once-daily long-acting muscarinic antagonist, in COPD patients. Respir Med. 2010;104:1482-1489.

52. Santus P, Radovanovic D, Di Marco F, Raccanelli R, Valenti V, Centanni S. Faster reduction in hyperinflation and improvement in lung ventilation inhomogeneity promoted by aclidinium compared to glycopyrronium in severe stable COPD patients. A randomized crossover study. Pulm Pharmacol Ther. 2015;35:42-49.
53. Villetti G, Bergamaschi M, Bassani F, et al. Pharmacological assessment of the duration of action of glycopyrrolate vs tiotropium and ipratropium in guinea-pig and human airways. Br J Pharmacol. 2006;148: 291-298.

54. Daley-Yates PT, Price AC, Sisson JR, et al. Beclomethasone dipropionate: absolute bioavailability, pharmacokinetics and metabolism following intravenous, oral, intranasal and inhaled administration in man. Br J Clin Pharmacol. 2001;51:400-409.

55. Leach CL, Davidson PJ, Hasselquist BE, et al. Lung deposition of hydrofluoroalkane-134a beclomethasone is greater than that of chlorofluorocarbon fluticasone and chlorofluorocarbon beclomethasone: a cross-over study in healthy volunteers. Chest. 2002;122:510-516.

56. Boobis AR. Comparative physicochemical and pharmacokinetic profiles of inhaled beclomethasone dipropionate and budesonide. Respir Med. 1998;92(Suppl B):2-6.

57. Hübner M, Hochhaus G, Derendorf H. Comparative pharmacology, bioavailability, pharmacokinetics, and pharmacodynamics of inhaled glucocorticosteroids. Immunol Allergy Clin North Am. 2005;25:469-488.

58. Bhatt SP, Soler X, Wang X, et al; COPDGene Investigators. Association between functional small airway disease and FEV1 decline in chronic obstructive pulmonary disease. Am J Respir Crit Care Med. 2016;194: 178-184.

59. Santus P, Radovanovic D, Di Marco S, et al. Effect of indacaterol on lung deflation improves cardiac performance in hyperinflated COPD patients: an interventional, randomized, double-blind clinical trial. Int J Chron Obstruct Pulmon Dis. 2015;10:1917-1923.

60. Santus P, Radovanovic D, Henchi S, et al. Assessment of acute bronchodilator effects from specific airway resistance changes in stable COPD patients. Respir Physiol Neurobiol. 2014;197:36-45.

61. Pecchiari M, Santus P, Radovanovic D, D'Angelo E. Acute effects of long-acting bronchodilators on small airways detected in COPD patients by single-breath N2 test and lung P-V curve. J Appl Physiol (1985). 2017; 123:1266-1275.

62. Calzetta L, Matera MG, Cazzola M. Pharmacological interaction between LABAs and LAMAs in the airways: optimizing synergy. Eur J Pharmacol. 2015;761:168-173.

63. Calzetta L, Matera MG, Cazzola M. Pharmacological mechanisms leading to synergy in fixed-dose dual bronchodilator therapy. Curr Opin Pharmacol. 2018;40:95-103.

64. Singh D, Schröder-Babo W, Cohuet G, et al; TRIDENT Study Investigators. The bronchodilator effects of extrafine glycopyrronium added to combination treatment with beclometasone dipropionate plus formoterol in COPD: a randomised crossover study (the TRIDENT study). Respir Med. 2016;114:84-90.

65. Calzetta L, Matera MG, Facciolo F, Cazzola M, Rogliani P. Beclomethasone dipropionate and formoterol fumarate synergistically interact in hyperresponsive medium bronchi and small airways. Respir Res. 2018;19:65.

66. Singh D, Corradi M, Spinola M, et al. Triple therapy in COPD: new evidence with the extrafine fixed combination of beclomethasone dipropionate, formoterol fumarate, and glycopyrronium bromide. Int J Chron Obstruct Pulmon Dis. 2017;12:2917-2928.

67. goldcopd.org. [homepage on the Internet]. From the Global Strategy for the Diagnosis, Management and Prevention of COPD, Global Initiative for Chronic Obstructive Lung Disease (GOLD); 2018 [updated 2018, cited May 18, 2018]. Available from: http://goldcopd.org. Accessed May 19, 2018.

68. Pearl Therapeutics, Inc. A study to assess the efficacy and safety of PT009 compared to PT005 on COPD exacerbations over a 52-week period in subjects with moderate to very severe COPD (Sophos). Available from: https://clinicaltrials.gov/ct2/show/NCT02727660. NLM identifier: NCT02727660. Accessed May 20, 2018.

69. Chiesi Farmaceutici S.p.A. Active controlled trial of CHF5993 pressurized metered-dose inhaler (pMDI) vs Symbicort ${ }^{\circledR}$ Turbuhaler $^{\circledR}$ in patients with chronic obstructive pulmonary disease (COPD) (TRIVERSYTI). Available from: https://clinicaltrials.gov/ct2/show/NCT03197818?term $=$ NCT03197818\&rank=1. NLM identifier: NCT03197818. Accessed May 20, 2018. 
70. Pearl Therapeutics, Inc. Study to assess the efficacy and safety of PT010 relative to PT003 and PT009 in subjects with moderate to very severe COPD (Ethos). Available from: https://clinicaltrials.gov/ ct2/show/NCT02465567. NLM identifier: NCT02465567. Accessed May 20, 2018.

71. Boehringer Ingelheim. Assessment in a real world setting of the effect of inhaled steroid-based triple therapy versus the combination of tiotropium and olodaterol on reducing chronic obstructive pulmonary disease (COPD) exacerbations [AIRWISE]. Available from: https:/clinicaltrials.gov/ct2/show/NCT03265145. NLM identifier: NCT03265145. Accessed May 20, 2018.
72. Chapman KR, Hurst JR, Frent S, et al. Long-term triple therapy deescalation to indacaterol/glycopyrronium in COPD patients (SUNSET): a randomized, double-blind, triple-dummy clinical trial. Am J Respir Crit Care Med. Epub 2018 May 20.

73. Calverley PMA. A light in the darkness? The FLAME trial, blood eosinophils, and chronic obstructive pulmonary disease. Am J Respir Crit Care Med. 2017;195:1125-1127.

International Journal of COPD

\section{Publish your work in this journal}

The International Journal of COPD is an international, peer-reviewed journal of therapeutics and pharmacology focusing on concise rapid reporting of clinical studies and reviews in COPD. Special focus is given to the pathophysiological processes underlying the disease, intervention programs, patient focused education, and self management protocols.

\section{Dovepress}

This journal is indexed on PubMed Central, MedLine and CAS. The manuscript management system is completely online and includes a very quick and fair peer-review system, which is all easy to use. Visit http://www.dovepress.com/testimonials.php to read real quotes from published authors.

Submit your manuscript here: http://www.dovepress.com/international-journal-of-chronic-obstructive-pulmonary-disease-journal 